\title{
Genetic variations in the CYP17A1 and NT5C2 genes are associated with a reduction in visceral and subcutaneous fat areas in Japanese women
}

\author{
Kikuko Hotta $^{1}$, Aya Kitamoto ${ }^{1}$, Takuya Kitamoto ${ }^{1}$, Seiho Mizusawa ${ }^{2}$, Hajime Teranishi ${ }^{2}$, Tomoaki Matsuo ${ }^{3}$, \\ Yoshio Nakata ${ }^{3}$, Hideyuki Hyogo ${ }^{4}$, Hidenori Ochi ${ }^{4}$, Takahiro Nakamura ${ }^{5}$, Seika Kamohara ${ }^{6}$, \\ Nobuyuki Miyatake ${ }^{7}$, Kazuaki Kotani ${ }^{8}$, Ryoya Komatsu ${ }^{9}$, Naoto Itoh ${ }^{10}$, Ikuo Mineo ${ }^{11}$, Jun Wada ${ }^{12}$, \\ Masato Yoneda ${ }^{13}$, Atsushi Nakajima ${ }^{13}$, Tohru Funahashi ${ }^{14}$, Shigeru Miyazaki ${ }^{15}$, Katsuto Tokunaga ${ }^{16}$, \\ Hiroaki Masuzaki ${ }^{17}$, Takato Ueno ${ }^{18}$, Kazuaki Chayama ${ }^{4}$, Kazuyuki Hamaguchi ${ }^{19}$, Kentaro Yamada $^{20}$, \\ Toshiaki Hanafusa $^{21}$, Shinichi Oikawa ${ }^{22}$, Hironobu Yoshimatsu ${ }^{23}$, Toshiie Sakata ${ }^{23}$, Kiyoji Tanaka ${ }^{3}$, \\ Yuji Matsuzawa ${ }^{8}$, Kazuwa Nakao ${ }^{1,24}$ and Akihiro Sekine ${ }^{1,2}$
}

Visceral fat accumulation has an important role in increasing the morbidity and mortality rates, by increasing the risk of developing several metabolic disorders, such as type 2 diabetes, dyslipidemia and hypertension. New genetic loci that are associated with increased systolic and diastolic blood pressures have been identified by genome-wide association studies in Caucasian populations. This study investigates whether single nucleotide polymorphisms (SNPs) that confer susceptibility to high blood pressure are also associated with visceral fat obesity. We genotyped 1279 Japanese subjects (556 men and 723 women) who underwent computed tomography for measuring the visceral fat area (VFA) and subcutaneous fat area (SFA) at the following SNPs: FGF5 rs16998073, CACNB2 rs11014166, C10orf107 rs1530440, CYP17A1 rs1004467, NT5C2 rs11191548, PLEKHA7 rs381815, ATP2B1 rs2681472 and rs2681492, ARID3B rs6495112, CSK rs1378942, PLCD3 rs12946454, and ZNF652 rs16948048. In an additive model, risk alleles of the CYP17A1 rs1004467 and NT5C2 rs 11191548 were found to be significantly associated with reduced SFA $(P=0.00011$ and 0.0016 , respectively). When the analysis was performed separately in men and women, significant associations of rs 1004467 (additive model) and rs 11191548 (recessive model) with reduced VFA $(P=0.0018$ and 0.0022 , respectively) and SFA $(P=0.00039$ and 0.00059 , respectively) were observed in women, but not in men. Our results suggest that polymorphisms in the CYP17A1 and NT5C2 genes influence a reduction in both visceral and subcutaneous fat mass in Japanese women.

Journal of Human Genetics (2012) 57, 46-51; doi:10.1038/jhg.2011.127; published online 10 November 2011

Keywords: computed tomography; CYP17A1; Japanese subjects; NT5C2; sexual dimorphism; subcutaneous fat area; visceral fat area

\section{INTRODUCTION}

Metabolic syndrome is a combination of multiple risk factors, including central obesity, impaired glucose tolerance, dyslipidemia and hypertension, which increases cardiovascular disease morbidity and mortality. ${ }^{1}$ Several studies have indicated that the intra-abdominal adipose tissue has a central role in metabolic syndrome, as the

${ }^{1}$ EBM Research Center, Kyoto University Graduate School of Medicine, Kyoto, Japan; ${ }^{2}$ Center for Genomic Medicine, Unit of Genome Informatics, Kyoto University Graduate School of Medicine, Kyoto, Japan; ${ }^{3}$ Graduate School of Comprehensive Human Sciences, University of Tsukuba, Tsukuba, Japan; ${ }^{4}$ Department of Medicine and Molecular Science, Division of Frontier Medical Science, Programs for Biomedical Research, Graduate School of Biomedical Sciences, Hiroshima University, Hiroshima, Japan; ${ }^{5}$ Laboratory for Mathematics, National Defense Medical College, Tokorozawa, Japan; ${ }^{6}$ Health Science University, Yamanashi, Japan; ${ }^{7}$ Department of Hygiene, Faculty of Medicine, Kagawa University, Kagawa, Japan; ${ }^{8}$ Department of Metabolic Medicine, Graduate School of Medicine, Osaka University, Osaka, Japan; ${ }^{9}$ Rinku General Medical Center, Osaka, Japan; ${ }^{10}$ Toyonaka Municipal Hospital, Osaka, Japan; ${ }^{11}$ Otemae Hospital, Osaka, Japan; ${ }^{12}$ Department of Medicine and Clinical Science, Okayama University Graduate School of Medicine, Dentistry and Pharmaceutical Sciences, Okayama, Japan; ${ }^{13}$ Division of Gastroenterology, Yokohama City University Graduate School of Medicine, Yokohama, Japan; ${ }^{14}$ Department of Metabolism and Atherosclerosis, Graduate School of Medicine, Osaka University, Osaka, Japan; ${ }^{15}$ Tokyo Postal Services Agency Hospital, Tokyo, Japan; ${ }^{16}$ Itami City Hospital, Hyogo, Japan; ${ }^{17}$ Division of Endocrinology and Metabolism, Second Department of Internal Medicine, University of the Ryukyus Faculty of Medicine, Okinawa, Japan; ${ }^{18}$ Research Center for Innovative Cancer Therapy, Kurume University, Kurume, Japan; ${ }^{19}$ Department of Community Health and Gerontological Nursing, Faculty of Medicine, Oita University, Oita, Japan; ${ }^{20}$ Division of Endocrinology and Metabolism, Department of Medicine, Kurume University, Kurume, Japan; ${ }^{21}$ Department of Internal Medicine (I), Osaka Medical College, Osaka, Japan; ${ }^{22}$ Division of Endocrinology and Metabolism, Department of Medicine, Nippon Medical School, Tokyo, Japan; ${ }^{23}$ Department of Internal Medicine 1, Faculty of Medicine, Oita University, Oita, Japan and ${ }^{24}$ Department of Medicine and Clinical Science, Kyoto University Graduate School of Medicine, Kyoto, Japan

Correspondence: Dr K Hotta, EBM Research Center, Kyoto University Graduate School of Medicine, Yoshida-Konoecho, Sakyo-ku, Kyoto 606-8501, Japan. E-mail: kikukoh@kuhp.kyoto-u.ac.jp

Received 1 August 2011; revised 4 October 2011; accepted 19 October 2011; published online 10 November 2011 
accumulated visceral adipose tissue leads to alterations in the plasma levels of adipocytokines, resulting in the development of dyslipidemia, hypertension and insulin resistance. ${ }^{2,3}$ Intra-abdominal fat accumulation (central adiposity) is determined by waist circumference, waisthip ratio, biological impedance or the visceral fat area (VFA) measured using computed tomography. ${ }^{1,4,5}$ There is abundant evidence that body fat distribution is influenced by genetic loci. ${ }^{6-8}$ Individual variation in waist-hip ratio is heritable, with heritability estimates ranging from 22 to $61 \%$. Recent genome-wide association studies (GWAS) showed that genetic loci were associated with waist circumference and waist-hip ratio in the Caucasian population. ${ }^{9,10}$ We previously reported that the rs1558902 and rs1421085 genotypes of the fat mass- and obesity-associated gene (FTO) were significantly associated with VFA, as well as with the subcutaneous fat area (SFA) and body mass index (BMI) in the Japanese population. ${ }^{11}$

Recent progress in GWAS has increased the number of known genetic susceptibility loci for obesity. ${ }^{12-16}$ We investigated the association between the single nucleotide polymorphisms (SNPs) underlying susceptibility to obesity and fat distribution (as determined by computed tomography), and found that rs7498665 in the SH2B adaptor protein 1 (SH2B1) gene was associated with VFA, uncovering the genetic background of central obesity. ${ }^{17}$

GWAS, and meta-analysis of GWAS, have identified various diseaseassociated genetic variations. ${ }^{18}$ Hypertension is one of the risk factors of metabolic syndrome and is considerably related to central obesity. Obesity-associated allele of rs1558902 and rs1421085 in the FTO gene were associated with hypertension, but not that of rs7498665 in the SH2B1 gene in the Japanese population. ${ }^{19}$ The genetic variations associated with hypertension have been identified by GWAS. ${ }^{20,21}$ In this study, we investigate whether the recently reported hypertension-related loci are also associated with VFA, which is another important factor responsible for metabolic syndrome.

\section{MATERIALS AND METHODS}

\section{Study subjects}

We enrolled 1279 Japanese subjects from outpatient clinics; these patients agreed to undergo computed tomography testing (in the supine position) to determine VFA and SFA values at the umbilical level (L4-L5), as previously reported. ${ }^{17}$ Both VFA and SFA values were calculated using the FatScan software program (N2system, Osaka, Japan). ${ }^{22}$ The patients visited the hospitals to undergo treatment for obesity and/or metabolic abnormalities, such as hypertension, dyslipidemia and type 2 diabetes. Patients with secondary obesity and obesity-related hereditary disorders were excluded from this study. Patients with disease (such as cancer, and renal, heart and hepatic failure), or under treatment (such as corticosteroid and chemotherapy) that strongly affects body weight, were also excluded. Athletes were also excluded from this study. Clinical data were recorded at the first visit to the hospital. The clinical characteristics of the subjects are summarized in Table 1. Metabolic syndrome and metabolic abnormalities were diagnosed according to the criteria released by the Japanese Committee for the Diagnostic Criteria of Metabolic Syndrome in April 2005., ${ }^{4,5}$ Written informed consent was obtained from each subject, and the protocol was approved by the ethics committee of each institution and by that of Kyoto University.

\section{DNA extraction and SNP genotyping}

Genomic DNA was extracted from the blood samples collected from each subject using the Genomix kit (Talent Srl, Trieste, Italy). We selected 12 SNPs that were previously identified as susceptibility loci for hypertension by GWAS in Caucasian populations, ${ }^{20,21}$ and constructed Invader probes (Third Wave Technologies, Madison, WI, USA) for each. The 12 selected SNPs were as follows: rs16998073 in the fibroblast growth factor 5 (FGF5) gene; rs11014166 in the calcium channel, voltage-dependent, $\beta-2$ subunit (CACNB2) gene; rs 1530440 in the chromosome 10 open reading frame 107 (C10orf107) gene;
Table 1 Clinical characteristics of the subjects

\begin{tabular}{lccc}
\hline & Men & Women & Total \\
\hline$n$ & 556 & 723 & 1279 \\
Age (years) & $49.4 \pm 12.2$ & $52.2 \pm 11.3$ & $51.0 \pm 11.8$ \\
BMI (kg m$\left.{ }^{-2}\right)$ & $30.2 \pm 6.1$ & $28.1 \pm 5.3$ & $29.0 \pm 5.8$ \\
VFA $\left(\mathrm{cm}^{2}\right)$ & $155.3 \pm 67.7$ & $99.8 \pm 53.6$ & $123.9 \pm 66.1$ \\
SFA $\left(\mathrm{cm}^{2}\right)$ & $206.7 \pm 108.6$ & $241.6 \pm 97.2$ & $226.5 \pm 103.7$ \\
Waist circumference $(\mathrm{cm})$ & $97.5 \pm 11.3$ & $91.8 \pm 10.3$ & $94.2 \pm 11.1$
\end{tabular}

Prevalence of metabolic disease

$\begin{array}{llll}\text { Dyslipidemia } & 293(53 \%) & 244(34 \%) & 537(42 \%) \\ \text { Hypertension } & 379(68 \%) & 452(63 \%) & 831(65 \%) \\ \text { Impaired fasting glucose } & 177(32 \%) & 176(24 \%) & 353(28 \%) \\ \text { Metabolic syndrome } & 248(45 \%) & 162(22 \%) & 410(32 \%)\end{array}$

Abbreviations: BMI, body mass index; SFA, subcutaneous fat area; VFA, visceral fat area. Data are represented as mean \pm s.d.

rs1004467 in the cytochrome P450, family 17, subfamily A, polypeptide 1 (CYP17A1) gene; rs11191548 in the $5^{\prime}$-nucleotidase, cytosolic II (NT5C2) gene; rs381815 in the pleckstrin homology domain containing, family A member 7 (PLEKHA7) gene; rs2681472 and rs2681492 in the ATPase, $\mathrm{Ca}^{2+}$ transporting, plasma membrane 1 (ATP2B1) gene; rs6495112 in the AT-rich interactive domain 3B (BRIGHT-like) (ARID3B) gene; rs1378942 in the c-src tyrosine kinase (CSK) gene; rs12946454 in the phospholipase C, delta 3 (PLCD3) gene; and rs16948048 in the zinc finger protein 652 (ZNF652) gene. The SNPs were genotyped using Invader assays, as previously described. ${ }^{23}$ The success rate of these assays was $>99.0 \%$.

\section{Statistical analysis}

For the additive model, we coded the genotypes as 0,1 or 2 depending on the number of copies of the risk alleles. For the recessive model, homozygosity with the risk allele was coded as 1 and the others were coded as 0 . Risk alleles refer to the hypertension-associated alleles, according to previous reports. ${ }^{20,21}$ Multiple linear regression analyses were performed to test the independent effect of the risk alleles on BMI, VFA and SFA, by taking into account the effects of other variables (that is, age and gender) that were assumed to be independent of the effect of each SNP. The values of BMI, VFA and SFA were logarithmically transformed before performing the multiple linear regression analysis. Differences in the quantities of anthropometric parameters among the different genotypes were assessed by the analysis of covariance, by taking into account the effects of other variables (that is, age and/or institute). Hardy-Weinberg equilibrium was assessed using the $\chi^{2}$-test. ${ }^{24}$ To test SNP $\times$ SNP epistasis, we used a linear regression model for each SNP1 and SNP2, and fit the model in the form of $Y=\beta_{0}+\beta_{1} \times \mathrm{SNP} 1+\beta_{2} \times \mathrm{SNP} 2+\beta_{3} \times \mathrm{SNP} 1 \times \mathrm{SNP} 2+\beta_{4} \times$ age $+\beta_{5} \times$ gender . Although we collected the samples at the region of Hondo (Kanto, Kinki, Chugoku and Kyushu; Supplementary Table 1), we performed Wright's F-statistics ${ }^{25}$ to evaluate the difference in the population structures of our sample using randomly selected 31 SNPs. We divided our samples into two groups (SFA $>208 \mathrm{~cm}^{2}$ and $\left.\leqslant 208 \mathrm{~cm}^{2}\right)$. Median of SFA $\left(208 \mathrm{~cm}^{2}\right)$ was used as a cut-off value. The results indicated that the population structure of the two groups were almost the same in view of a very small $F_{\mathrm{ST}}$ value between both the groups (mean $F_{\mathrm{ST}}=0.00023$ ). Statistical analysis was performed using R software (http://www.r-project.org/). P-values were assessed with a Bonferroni correction and $P<0.0042(0.05 / 12)$ was considered statistically significant.

\section{RESULTS}

The clinical characteristics and genotypes of the subjects are shown in Tables 1 and 2, respectively. All the SNPs were in Hardy-Weinberg equilibrium and the minor allele frequencies did not diverge from those reported in the HapMap database. The BMI, VFA and SFA values for each SNP genotype are reported in Table 3. Multiple linear regression analyses of the anthropometric parameters with respect to the 12 analyzed SNPs are shown in Table 4. The A-allele of rs1004467 
Table 2 Genotypic characteristics of the subjects

\begin{tabular}{|c|c|c|c|c|c|c|c|}
\hline$S N P I D$ & $\mathrm{CHR}$ & Position (Build 36.3) & Nearby gene & Allele 1/2 & BP-associated allele & Genotype & HWE P-value \\
\hline rs11014166 & 10 & 18748804 & CACNB2 & T/A & A & 4/124/1151 & 0.73 \\
\hline rs1004467 & 10 & 104584497 & CYP17A1 & $A / G$ & A & $559 / 567 / 153$ & 0.62 \\
\hline rs11191548 & 10 & 104836168 & NT5C2 & $\mathrm{T} / \mathrm{C}$ & $T$ & $675 / 504 / 100$ & 0.66 \\
\hline rs381815 & 11 & 16858844 & PLEKHA7 & $\mathrm{C} / \mathrm{T}$ & $\mathrm{T}$ & $842 / 381 / 56$ & 0.13 \\
\hline rs6495112 & 15 & 72619851 & $A R I D 3 B$ & $\mathrm{~A} / \mathrm{C}$ & A & $530 / 575 / 173$ & 0.39 \\
\hline rs1378942 & 15 & 72864420 & CSK & $\mathrm{A} / \mathrm{C}$ & c & 49/410/817 & 0.78 \\
\hline rs12946454 & 17 & 40563647 & PLCD3 & $T / A$ & T & $34 / 343 / 901$ & 0.84 \\
\hline rs16948048 & 17 & 44795465 & ZNF652 & $\mathrm{G} / \mathrm{A}$ & G & $18 / 326 / 935$ & 0.08 \\
\hline
\end{tabular}

Abbreviations: BP, blood pressure; CHR, chromosome; HWE, Hardy-Weinberg equilibrium.

Table 3 Mean BMI, VFA and SFA for 12 blood pressure risk variants

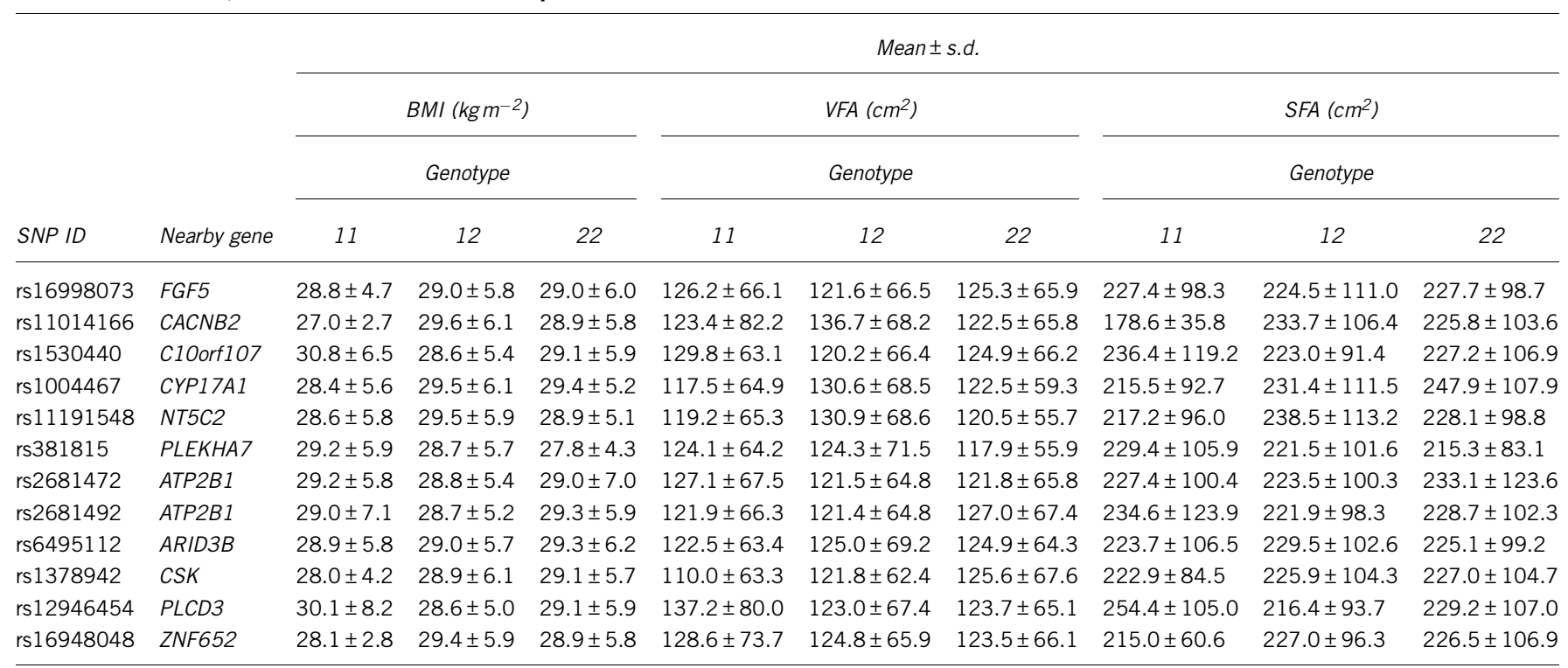

Abbreviations: BMI, body mass index; SFA, subcutaneous fat area; SNP, single nucleotide polymorphism; VFA, visceral fat area.

11, allele1/allele1; 12, allele1/allele2; 22, allele2/allele2. Allele 1 and allele 2 of each SNP is indicated in Table 2.

in the CYP17A1 gene was significantly associated with reduced BMI $(P=0.0018)$. The other SNPs were not significantly associated with BMI. No SNP was significantly associated with VFA. The A-allele of rs1004467 in the CYP17A1 and the T-allele of rs11191548 in the NT5C2 gene were significantly associated with reduced SFA. These SNPs are in linkage disequilibrium, as reported in the HapMap database $\left(D^{\prime}=0.98, r^{2}=0.71\right)$, and the A-allele of rs1004467 and T-allele of rs11191548 are reported to be risk alleles for increased blood pressure. $^{20,21}$

BMI, VFA and SFA are known to be affected by gender; therefore, we compared rs1004467 and rs11191548 alleles with anthropometric parameters (BMI, VFA and SFA) in men and women independently (Table 5). Associations of both SNPs with VFA $(P=0.0018$ and $P=0.0043)$ and SFA $(P=0.00039$ and $P=0.0021)$ in women were significant, except the association of T-allele of rs11191548 with VFA. The VFA and SFA values of the rs11191548 genotype suggest that the recessive model would be the best-fitted model both in men and women. By using the recessive model, results revealed significant associations of the rs11191548 genotype with VFA $(P=0.0022)$ and SFA in women $(P=0.00059)$. These SNPs did not show any association with VFA or SFA in men, suggesting that they exhibit sexual dimorphism, as has been suggested in a recent report. ${ }^{26}$ As both rs1004467 and rs11191548 were associated with a reduction in both VFA and SFA, we examined the association of these SNPs with total fat area. The SNPs were significantly associated with total fat area $(P=0.00012$ at rs1004467, $P=0.00052$ at $\mathrm{rs} 11191548$ in additive model) in women, but not in men, suggesting that risk allele for high blood pressure of these SNPs are associated with reduced adiposity in women. The very small mean $F_{\mathrm{ST}}$ value $(0.00023)$ indicated no population structure in our subjects. As we collected the samples from nine institutes in four regions of Japan (Supplementary Table 1), we tested multiple linear regression analysis with age and institute as explanatory variables in men and women. Very similar results were observed. In additive model, significant associations of the 
Table 4 Relationship between blood pressure-associated loci and adiposity measures

\begin{tabular}{|c|c|c|c|c|c|c|c|c|c|c|}
\hline \multirow[b]{2}{*}{$S N P I D$} & \multirow[b]{2}{*}{ Nearby gene } & \multicolumn{3}{|c|}{$B M I$} & \multicolumn{3}{|c|}{$V F A$} & \multicolumn{3}{|c|}{ SFA } \\
\hline & & $\beta$ & s.e. & P-value & $\beta$ & s.e. & $\mathrm{P}$-value & $\beta$ & s.e. & P-value \\
\hline rs16998073 & FGF5 & -0.002 & 0.003 & 0.55 & -0.003 & 0.010 & 0.78 & -0.010 & 0.008 & 0.22 \\
\hline rs11014166 & CACNB2 & -0.005 & 0.007 & 0.48 & -0.043 & 0.021 & 0.043 & -0.008 & 0.017 & 0.63 \\
\hline rs1530440 & C10orf107 & -0.002 & 0.004 & 0.71 & 0.010 & 0.014 & 0.48 & -0.005 & 0.011 & 0.64 \\
\hline rs1004467 & CYP17A1 & -0.010 & 0.003 & 0.0018 & -0.022 & 0.010 & 0.027 & -0.030 & 0.008 & 0.00011 \\
\hline rs11191548 & NT5C2 & -0.008 & 0.003 & 0.015 & -0.019 & 0.011 & 0.078 & -0.026 & 0.008 & 0.0016 \\
\hline rs381815 & PLEKHA7 & -0.007 & 0.004 & 0.046 & -0.004 & 0.012 & 0.76 & -0.015 & 0.009 & 0.10 \\
\hline rs2681472 & ATP2B1 & 0.002 & 0.003 & 0.43 & 0.006 & 0.010 & 0.52 & 0.005 & 0.008 & 0.49 \\
\hline rs2681492 & ATP2B1 & 0.003 & 0.003 & 0.34 & 0.006 & 0.010 & 0.54 & 0.006 & 0.008 & 0.40 \\
\hline rs6495112 & $A R I D 3 B$ & -0.002 & 0.003 & 0.45 & -0.004 & 0.010 & 0.65 & -0.007 & 0.008 & 0.36 \\
\hline rs1378942 & CSK & 0.005 & 0.004 & 0.20 & 0.010 & 0.012 & 0.40 & 0.005 & 0.009 & 0.61 \\
\hline rs12946454 & PLCD3 & -0.003 & 0.004 & 0.39 & 0.009 & 0.013 & 0.50 & -0.011 & 0.010 & 0.28 \\
\hline rs16948048 & ZNF652 & 0.005 & 0.004 & 0.30 & 0.008 & 0.014 & 0.57 & 0.005 & 0.011 & 0.67 \\
\hline
\end{tabular}

Abbreviations: BMI, body mass index; SFA, subcutaneous fat area; SNP, single nucleotide polymorphism; VFA, visceral fat area.

Data were derived from a linear regression analysis. The values of BMI, VFA and SFA were logarithmically transformed. Logarithmically transformed BMI, VFA and SFA were adjusted for age and gender. Tested alleles are risk alleles of increased blood pressure.

Table 5 Relationship between rs1004467 and rs11191548, and adiposity in men and women

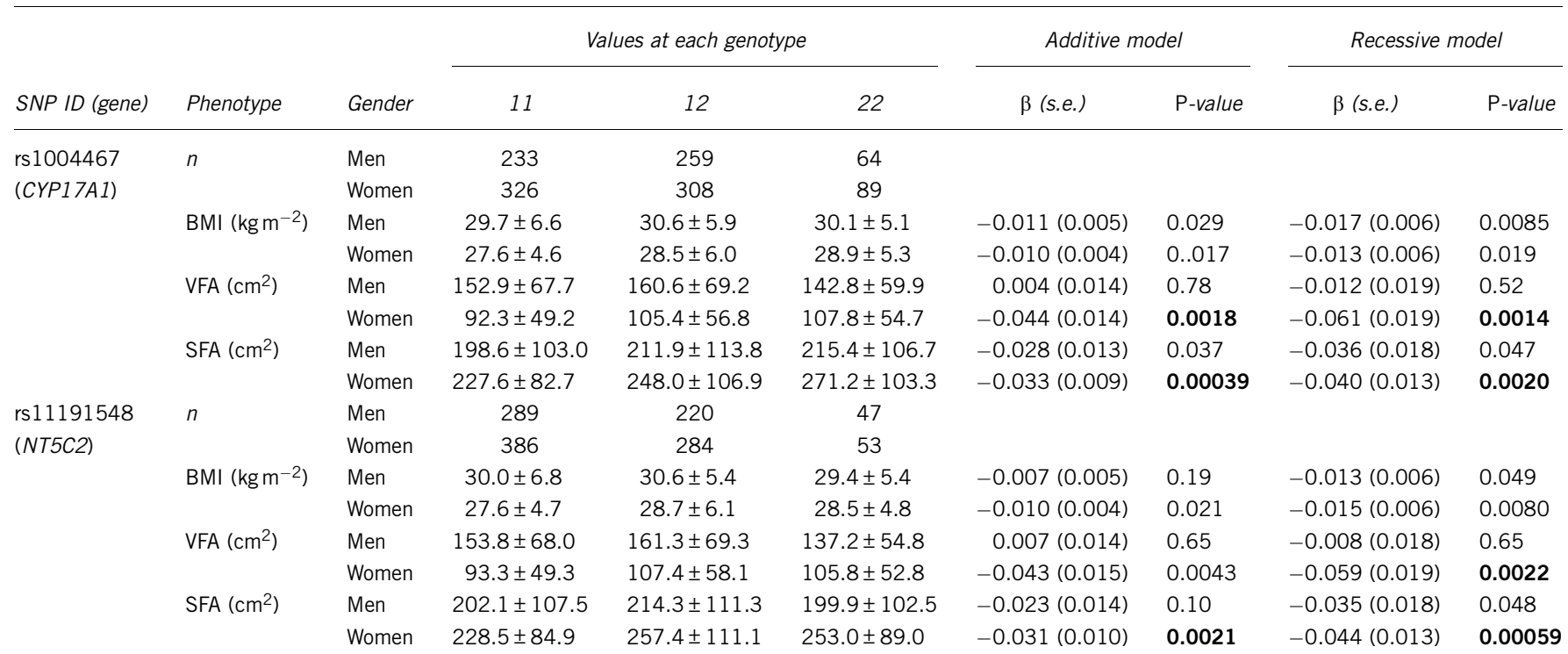

Abbreviations: BMI, body mass index; SFA, subcutaneous fat area; SNP, single nucleotide polymorphism; VFA, visceral fat area.

Values are shown as the mean \pm s.d. Data were derived from a linear regression analysis. The values of BMI, VFA and SFA were logarithmically transformed. Logarithmically transformed BMI, VFA and SFA were adjusted for age. Tested alleles (allele1 at both SNPs) are risk alleles of increased blood pressure.

rs1004467 and rs11191548 genotype with VFA $(P=0.0015$ and 0.0011 , respectively) and SFA ( $P=0.00021$ and 0.00062 , respectively) were observed in women (Supplementary Table 2). Statistical analysis using analysis of covariance indicated significant associations of the rs1004467 and rs11191548 genotype with VFA $(P=0.0020$ and 0.0015 , respectively) and SFA ( $P=0.00033$ and 0.00042 , respectively) in women (Supplementary Table 2). As some diabetes medications have an effect on adiposity, ${ }^{27}$ we performed the analysis excluding 147 type 2 diabetic patients treated with sulfonylureas, biguanides and thiazolidinediones We found the similar significant associations of the rs1004467 and rs11191548 genotype with VFA and SFA in women (Supplementary Table 3).

We have reported that rs1558902 in the FTO gene is associated with both VFA and SFA, ${ }^{11}$ and that rs7498665 in the SH2B1 gene is associated with VFA. ${ }^{17}$ Thus, we examined SNP $\times$ SNP epistasis in men, women and all subjects. The combination of rs1004467 and rs7498665 exhibited no epistatic effect on VFA in men $(P=0.43)$, women $(P=0.86)$ or all subjects $(P=0.76)$. The combination of rs1004467 and rs1558902 did not show epistatic effect on VFA in men $(P=0.99)$, women $(P=0.53)$ or all subjects $(P=0.60)$, or on SFA in men $(P=0.63)$, women $(P=0.83)$ or all subjects $(P=0.89)$.

Among the SNPs tested in this study, rs16998073 in the FGF5 gene and rs11191548 in the NT5C2 gene were associated with increased systolic blood pressure $(P<0.05)$. Rs11191548 in the NT5C2 gene were also associated with hypertension $(P<0.05)$. We could replicate the association between blood pressure and the above two SNPs that were reported to be strongly associated with blood pressure in the Japanese population (Supplementary Table 4). ${ }^{28}$ 


\section{DISCUSSION}

In this study, we showed that the A-allele of rs1004467 in the CYP17A1 and the T-allele of rs11191548 in the NT5C2 gene were significantly associated with reduced VFA, SFA and total fat area in women. Association of T-allele of rs11191548 in the NT5C2 gene with increased systolic blood pressure and hypertension was replicated in our sample, as reported previously. ${ }^{28}$ Our hypothesis was that these risk alleles would be associated with increased VFA and/or SFA as increased adiposity is a risk for hypertension; $;^{4,5}$ however, these alleles affected decreased adiposity. The associations between SNPs and increased blood pressure/hypertension were evaluated after being adjusted for BMI, age and gender. Thus, the SNPs associated with visceral fat obesity-related and gender-dependent hypertension would be excluded in the screening stage. Indeed, recent analysis has shown that genetic variation near insulin receptor substrate 1 (IRS1) is associated with reduced adiposity and an impaired metabolic profile. ${ }^{29}$ Thus, it is likely that rs1004467 and rs11191548 are associated with reduced VFA and SFA, as well as with hypertension in women.

The SNPs rs1004467 and rs11191548 were not associated with BMI in men or women, as reported for rs2943650 near IRS $1 .{ }^{29}$ As BMI represents both fat and lean body mass, our observation suggests that these SNPs influence a reduction in VFA and SFA, or influence an increased percentage of lean body mass. The significant associations of rs1004467 and rs1191548 with reduced VFA and SFA were observed in women, but not in men. The rs1004467 SNP is located in the intron of the CYP17A1 gene. CYP17A1 is involved in the biosynthesis of glucocorticoids, mineral corticoids, androgens and estrogens. $^{30}$ The rs1004467 risk allele may reflect differences in CYP17A1 gene expression that alter the biosynthesis of steroid hormones, leading to hypertension and reduced adiposity in women. The region of linkage disequilibrium that includes rs1004467 and rs11191548 contains a couple of genes in addition to CYP17A1: NT5C2, arsenic ( +3 oxidation state) methyltransferase (AS3MT) and cyclin M2 (CNNM2). NT5C2 is a cytosolic IMP/ GMP selective $5^{\prime}$-nucleotidase and involved in nucleic acids or DNA synthesis. ${ }^{31} \mathrm{CNNM} 2$ (ancient conserved domain protein, ACDP2) is a transporter of magnesium, which is required for the catalytic activity of numerous metalloenzymes. ${ }^{32}$ Thus, these genes would be important for metabolism in adipocyte hyperplasia and hypertrophy. Further investigation is warranted to elucidate the functional SNPs and susceptibility genes.

We have previously reported that FTO rs 1558902 is associated with VFA and SFA, and that SH2B1 rs7498665 is associated with VFA. ${ }^{11,17}$ Epistasis, or gene-gene interaction, has recently received much attention in human genetics. ${ }^{33}$ In this study, the effect of these SNPs on VFA and SFA was additive, and an epistatic effect was not observed.

In summary, we showed that CYP17A1 rs1004467 and NT5C2 rs11191548 SNPs are significantly associated with both reduced VFA and SFA in women. Our results suggest that the region encompassing CYP17A1 to NT5C2 has a role in reducing visceral and subcutaneous fat mass. However, these results require confirmation in other populations.

\section{CONFLICT OF INTEREST}

The authors declare no conflict of interest.

\section{ACKNOWLEDGEMENTS}

This work was supported by a Grant-in-Aid from the Ministry of Education, Science, Sports, and Culture of Japan (21591186 to Hotta, K., 23701082 to Kitamoto, T., and 23791027 to Kitamoto, A.), and by the Mitsui Life Science Social Welfare Foundation.
1 Carr, D. B., Utzschneider, K. M., Hull, R. L., Kodama, K., Retzlaff, B. M., Brunzell, J. D. et al. Intra-abdominal fat is a major determinant of the National Cholesterol Education Program Adult Treatment Panel III criteria for the metabolic syndrome. Diabetes 53, 2087-2094 (2004).

2 Matsuzawa, Y. Therapy insight: adipocytokines in metabolic syndrome and related cardiovascular disease. Nat. Clin. Pract. Cardiovasc. Med. 3, 35-42 (2006).

3 Hotta, K., Funahashi, T., Bodkin, N. L., Ortmeyer, H. K., Arita, Y., Hansen, B. C. et al. Circulating concentrations of the adipocyte protein adiponectin are decreased in parallel with reduced insulin sensitivity during the progression to type 2 diabetes in rhesus monkeys. Diabetes 50, 1126-1133 (2001).

4 Mastuzawa, Y. Metabolic syndrome-definition and diagnostic criteria in Japan. J. Atheroscler. Thromb. 12, 301 (2005).

5 Arai, H., Yamamoto, A., Matsuzawa, Y., Saito, Y., Yamada, N., Oikawa, S. et al. Prevalence of metabolic syndrome in the general Japanese population in 2000. J. Atheroscler. Thromb. 13, 202-208 (2006).

6 Selby, J. V., Newman, B., Quesenberry, C. P. Jr., Fabsitz, R. R., Carmelli, D., Meaney, F. J. et al. Genetic and behavioral influences on body fat distribution. Int. J. Obes. 14, 593-602 (1990).

7 Rose, K. M., Newman, B., Mayer-Davis, E. J. \& Selby, J. V. Genetic and behavioral determinants of waist-hip ratio and waist circumference in women twins. Obes. Res. $\mathbf{6}$, 383-392 (1998).

8 Souren, N. Y., Paulussen, A. D., Loos, R. J., Gielen, M., Beunen, G., Fagard, R. et al. Anthropometry, carbohydrate and lipid metabolism in the East Flanders Prospective Twin Survey: heritabilities. Diabetologia 50, 2107-2116 (2007).

9 Lindgren, C. M., Heid, I. M., Randall, J. C., Lamina, C., Steinthorsdottir, V., Qi, L. et al. Genome-wide association scan meta-analysis identifies three loci influencing adiposity and fat distribution. PLoS Genet. 5, e1000508 (2009).

10 Heard-Costa, N. L., Zillikens, M. C., Monda, K. L., Johansson, A., Harris, T. B., Fu, M. et al. NRXN3 is a novel locus for waist circumference: a genome-wide association study from the CHARGE Consortium. PLoS Genet. 5, e1000539 (2009).

11 Hotta, K., Nakamura, M., Nakamura, T., Matsuo, T., Nakata, Y., Kamohara, S. et al. Polymorphisms in NRXN3, TFAP2B, MSRA, LYPLAL1, FTO and MC4R and their effect on visceral fat area in the Japanese population. J. Hum. Genet. 55, 738-742 (2010).

12 Tanabe, A., Yanagiya, T., lida, A., Saito, S., Sekine, A., Takahashi, A. et al. Functional single-nucleotide polymorphisms in the secretogranin III (SCG3) gene that form secretory granules with appetite-related neuropeptides are associated with obesity. J. Clin. Endocrinol. Metab. 92, 1145-1154 (2007).

13 Yanagiya, T., Tanabe, A., Iida, A., Saito, S., Sekine, A., Takahashi, A. et al. Association of single-nucleotide polymorphisms in MTMR9 gene with obesity. Hum. Mol. Genet. 16, 3017-3026 (2007).

14 Thorleifsson, G., Walters, G. B., Gudbjartsson, D. F., Steinthorsdottir, V., Sulem, P., Helgadottir, A. et al. Genome-wide association yields new sequence variants at seven loci that associate with measures of obesity. Nat. Genet. 41, 18-24 (2009).

15 Willer, C. J., Speliotes, E. K., Loos, R. J., Li, S., Lindgren, C. M., Heid, I. M. et al. Six new loci associated with body mass index highlight a neuronal influence on body weight regulation. Nat. Genet. 41, 25-34 (2009).

16 Meyre, D., Delplanque, J., Chèvre, J. C., Lecoeur, C., Lobbens, S., Gallina, S. et al. Genome-wide association study for early-onset and morbid adult obesity identifies three new risk loci in European populations. Nat. Genet. 41, 157-159 (2009).

17 Hotta, K., Kitamoto, T., Kitamoto, A., Mizusawa, S., Matsuo, T., Nakata, Y. et al. Computed tomography analysis of the association between SH2B1 rs7498665 single-nucleotide polymorphism and visceral fat area. J. Hum. Genet. 56, 716-719 (2011)

18 Hindorff, L. A., Sethupathy, P., Junkins, H. A., Ramos, E. M., Mehta, J. P., Collins, F. S. et al. Potential etiologic and functional implications of genome-wide association loci for human diseases and traits. Proc. Natl Acad. Sci. USA 106, 9362-9367 (2009).

19 Hotta, K., Kitamoto, T., Kitamoto, A., Mizusawa, S., Matsuo, T., Nakata, Y. et al. Association of variations in the FTO, SCG3 and MTMR9 genes with metabolic syndrome in a Japanese population. J. Hum. Genet. 56, 647-651 (2011).

20 Levy, D., Ehret, G. B., Rice, K., Verwoert, G. C., Launer, L. J., Dehghan, A. et al. Genome-wide association study of blood pressure and hypertension. Nat. Genet. 41, 677-687 (2009).

21 Newton-Cheh, C., Johnson, T., Gateva, V., Tobin, M. D., Bochud, M., Coin, L. et al. Genome-wide association study identifies eight loci associated with blood pressure. Nat. Genet. 41, 666-676 (2009).

22 Yoshizumi, T., Nakamura, T., Yamane, M., Islam, A. H., Menju, M., Yamasaki, K. et al. Abdominal fat: standardized technique for measurement at CT. Radiology 211, 283-286 (1999).

23 Ohnishi, Y., Tanaka, T., Ozaki, K., Yamada, R., Suzuki, H. \& Nakamura, Y. A highthroughput SNP typing system for genome-wide association studies. J. Hum. Genet. 46, 471-477 (2001).

24 Nielsen, D. M., Ehm, M. G. \& Weir, B. S. Detecting marker-disease association by testing for Hardy-Weinberg disequilibrium at a marker locus. Am. J. Hum. Genet. 63, 1531-1540 (1998).

25 Wright, S. The genetical structure of populations. Ann. Eugen. 15, 323-354 (1951).

26 Heid, I. M., Jackson, A. U., Randall, J. C., Winkler, T. W., Qi, L., Steinthorsdottir, V. et al. Meta-analysis identifies 13 new loci associated with waist-hip ratio and reveals sexual dimorphism in the genetic basis of fat distribution. Nat. Genet. 42, 949-960 (2010). 
27 Mitri, J. \& Hamdy, O. Diabetes medications and body weight. Expert Opin. Drug Saf. 8, 573-584 (2009).

28 Takeuchi, F., Isono, M., Katsuya, T., Yamamoto, K., Yokota, M., Sugiyama, T. et al. Blood pressure and hypertension are associated with 7 loci in the Japanese population. Circulation 121, 2302-2309 (2010).

29 Kilpeläinen, T. O., Zillikens, M. C., Stančákova, A., Finucane, F. M., Ried, J. S., Langenberg, C. et al. Genetic variation near IRS1 associates with reduced adiposity and an impaired metabolic profile. Nat. Genet. 43, 753-760 (2011).
30 Gilep, A. A., Sushko, T. A. \& Usanov, S. A. At the crossroads of steroid hormone biosynthesis: the role, substrate specificity and evolutionary development of CYP17. Biochim. Biophys. Acta 1814, 200-209 (2011).

31 Itoh, R. IMP-GMP 5'-nucleotidase. Comp. Biochem. Physiol. B 105, 13-19 (1993).

32 Goytain, A. \& Quamme, G. A. Functional characterization of ACDP2 (ancient conserved domain protein), a divalent metal transporter. Physiol. Genomics 22, 382-389 (2005).

33 Cordell, H. Detecting gene-gene interactions that underlie human diseases. Nat. Rev. Genet. 10, 392-404 (2009).

Supplementary Information accompanies the paper on Journal of Human Genetics website (http://www.nature.com/jhg) 